\title{
Copolymerization of Carbon Dioxide with Optically Active Propylene Oxide
}

\author{
Shohei Inoue, Hideomi Koinuma, and Teiji Tsuruta \\ Department of Synthetic Chemistry, Faculty of \\ Engineering, University of Tokyo, Japan.
}

(Received October 31, 1970)

\begin{abstract}
Alternate copolymerization of carbon dioxide with optically active propylene oxide using a diethylzinc-water system as catalyst was investigated. The hydrolysis of the copolymer, poly(propylene carbonate), followed by subsequent treatment with $p$-nitrobenzoyl chloride, transformed the epoxide unit of the copolymer into the form of propylene glycol bis- $p$-nitrobenzoate. The determination of the optical activity of this ester led to the conclusion that the ring opening of propylene oxide takes place predominantly at the methylene-oxygen linkage in the copolymerization, which suggests the anionic nature of the reaction. The optical rotatory behavior of the copolymer is also described.
\end{abstract}

KEY WORDS Carbon Dioxide / Copolymerization / Diethylzinc / Optical Activity/Poly(propylene carbonate)/Propylene Oxide/ Ring Opening / Alternation /

As we have already reported, carbon dioxide copolymerizes alternately with alkylene oxide with the diethylzinc-water $(1: 1)$ system as catalyst to give a polycarbonate of high molecular weight ${ }^{1,2}$. In the copolymerization with propylene oxide, the epoxide has two different linkages to be cleaved, i.e., methylene-oxygen and methine-oxygen bonds. The mode of the ring opening of propylene oxide reflects the nature of the reaction in which the epoxide takes part; usually, under basic conditions, the methylene-oxygen linkage is predominantly cleaved whereas under acidic conditions ring opening at the methine-oxygen bond becomes relatively important.

In the homopolymerization of propylene oxide with organozinc catalyst systems, we have suggested predominant cleavage at methylene--oxygen bond on the basis of the optical activity of the polymer formed from the optically active monomer ${ }^{3}$.

In the copolymerization of carbon dioxide with propylene oxide, poly(propylene carbonate) formed may be readily cleaved by hydrolysis to a propylene glycol unit, and the optical purity of the unit in the copolymer from the optically active monomer may be directly determined.
In order to relate the optical purity of the epoxide unit in the copolymer to the mode of the ring opening, it is necessary to know whether the configuration of the asymmetric carbon atom is retained or inverted when the methine-oxygen bond is cleaved. We therefore studied the stereochemistry of the copolymerization of carbon dioxide with cyclohexene oxide using the diethylzinc-water $(1: 1)$ system, and found that this cis-epoxide is specifically converted to trans structure when incorporated into the copolymer. It was thus concluded that the configuration of the carbon atom where the ring opening takes place was inverted in the copolymerization ${ }^{4}$.

On this basis, the results obtained in the copolymerization of carbon dioxide with optically active propylene oxide is discussed in this paper. The optical rotatory behavior of the copolymer is also described.

\section{RESULTS AND DISCUSSION}

Copolymerization of $R-(+)$-Propylene Oxide with Carbon Dioxide

Copolymerization of $\mathrm{R}-(+)$-propylene oxide $(10.3 \mathrm{~g})$ with carbon dioxide $\left(45 \mathrm{~kg} / \mathrm{cm}^{2}\right)$ with the diethylzinc-water $(1: 0.9)$ system in dioxane 
at room temperature for $97 \mathrm{hr}$ produced polymer weighting $1.67 \mathrm{~g}$ which included an amount of $1.29 \mathrm{~g}$ insoluble in methanol. The methanol insoluble copolymer had an intrinsic viscosity of $0.36 \mathrm{~d} l / g$ (measured in benzene solution at $30^{\circ} \mathrm{C}$ ) and spectroscopic analyses confirmed it to be a completely alternate copolymer, i.e., poly(propylene carbonate) $-\left(-\mathrm{CH}_{2}-\mathrm{CH}\left(\mathrm{CH}_{3}\right)-\mathrm{O}-\right.$ $\mathrm{CO}-\mathrm{O}-)_{\bar{x}}$. For example, its NMR spectrum $(60 \mathrm{MHz})$ measured in benzene solution at $30^{\circ} \mathrm{C}$ showed only three peaks at $\tau=8.7,5.8$, and 5.0 which according to our previous report ${ }^{1,2}$ are assignable respectively to methyl, methylene, and methine protons in poly(propylene carbonate).
No signal was observed around $\tau=6.5$ due to the continuing propylene oxide units. The IR spectrum was exactly the same as that of the methanol-insoluble part of the copolymer from carbon dioxide and racemic propylene oxide ${ }^{2}$.

Mode of Ring Opening in the Copolymerization

In order to determine the extent of methylene -oxygen scission of propylene oxide during the copolymerization, propylene oxide unit in the $\mathrm{R}-(+)$-propylene oxide-carbon dioxide copolymer was isolated in the form of propylene glycol bis- $p$-nitrobenzoate (sample I) in accordance with the following process:

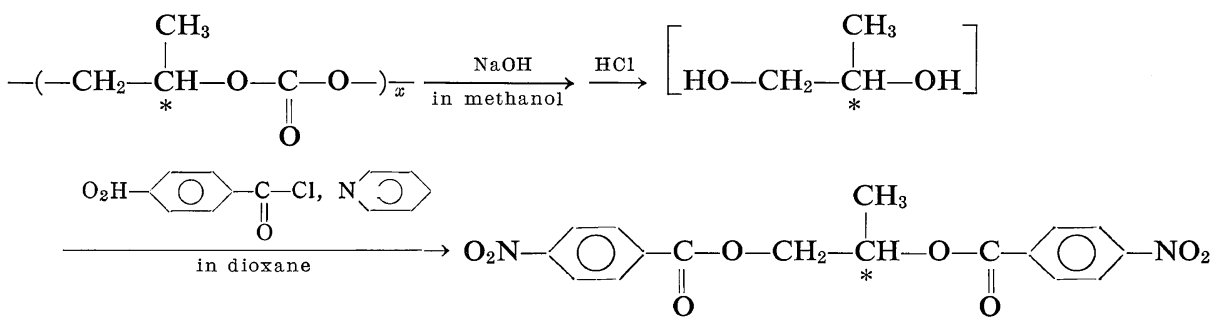

A racemic sample was also prepared from racemic propylene oxide-carbon dioxide copolymer using the same process as employed for the optically active copolymer. These reactions proceeded smoothly, and overall yield of the product was quite high $(>70 \%)$.

Table I shows the specific optical rotation at $589 \mathrm{~m} \mu\left([\alpha]_{\mathrm{D}}\right)$ of the product and compares it with the authentic sample (II) prepared from

Table I. Optical rotation of propylene bis-p-nitrobenzoate

\begin{tabular}{|c|c|c|c|}
\hline $\begin{array}{c}\text { Sample } \\
\text { no. }\end{array}$ & Source & $\begin{array}{c}{[\alpha]_{\mathrm{D}}^{25}} \\
(\text { benzene })^{\mathrm{a}}\end{array}$ & $\mathrm{mp},{ }^{\circ} \mathrm{C}$ \\
\hline & $\begin{array}{l}\text { Copolymer from } \\
\mathrm{R}-(+) \text {-propylene } \\
\text { oxide of }[\alpha]_{\mathrm{D}} \\
+13.2 \text { (neat) }\end{array}$ & -18.2 & $112-119$ \\
\hline II & $\begin{array}{l}\text { R-(-)-Propylene } \\
\text { glycol, }[\alpha]_{\mathrm{D}} \\
-15.8 \text { (neat) }\end{array}$ & -27.9 & $112-114$ \\
\hline III & $\begin{array}{l}\text { Copolymer from } \\
\text { RS-propylene oxide }\end{array}$ & - & $126-128$ \\
\hline
\end{tabular}

a $c, 1.0 \mathrm{~g} / \mathrm{d} l$.

b A mixture of samples II and III $(70: 30)$ has a melting range of $112-118^{\circ} \mathrm{C} .[\alpha]_{\mathrm{D}}^{25}-19.6^{\circ}$.
$\mathrm{R}$-(-)-propylene glycol and p-nitrobenzoyl chloride.

In order to estimate the optical purity of the propylene oxide unit in the copolymer, the $[\alpha]_{D}$ value of optically pure propylene oxide should be known. The $[\alpha]_{D}^{25}$ value for optically pure $\mathbf{R}-(+)$-propylene oxide so far reported is at least $+8.9^{\circ}$ in chloroform ${ }^{5}$. Since a propylene oxide with $[\alpha]_{D}^{24}+7.05^{\circ}$ in chloroform has $[\alpha]_{D}^{24}$ value of $+14.5^{\circ}$ in ether ${ }^{6}$ or neat, the optically pure propylene oxide is calculated, on the basis of the data given in Table $\mathrm{I}$, to give $p$-nitrobenzoate with $[\alpha]_{\mathrm{D}}^{25}-25.2^{\circ}(-18.2 \times 8.9 /$ $7.05 \times 14.5 / 13.2)$ through the copolymerization, hydrolysis and esterification. On the other hand, the $[\alpha]_{D}^{26}$ value for optically pure $\mathrm{R}-(-)$-propylene glycol is reported to be $-15.9^{\circ}$ (neat) ${ }^{7}$. Then the $[\alpha]_{\mathrm{D}}^{25}$ value of sample II in Table I, $-27.9^{\circ}$, can be compared with the calculated value, $-25.2^{\circ}$, for the sample obtained from optically pure propylene oxide by the same procedure as for sample I.

Since the reaction of glycol with $p$-nitrobenzoyl chloride is common in the preparation of samples I and II, the difference between the above two $[\alpha]_{D}$ values should be due to the 
racemization in the copolymerization and/or in the hydrolysis of the copolymer. As for the latter, no racemization is considered to occur in the hydrolysis under present conditions from our study on the copolymer of carbon dioxide with cyclochexene oxide and its model compounds ${ }^{4}$. Observed racemization, 9.6\% (27.9-25.2/27.9), is thus considered ascribable to that in the copolymerization reaction itself. Since every methine-oxygen scission is accompanied by the inversion of the configuration of the asymmetric carbon atom as described previously, racemization of $9.6 \%$ corresponds to $4.8 \%$ methineoxygen cleavage or $95.2 \%$ methylene-oxygen cleavage of the propylene oxide ring.

It is interesting to note almost the same degree of methylene-oxygen cleavage observed in the homopolymerization of propylene oxide by organozinc catalyst systems ${ }^{3}$. In the homopolymerization, almost the same percentage of methylene-oxygen scission was observed also in the reaction by potassium hydroxide, a typical anionic catalyst. It should be also mentioned here that 1,4-epoxycyclohexane ${ }^{4}$, tetrahydrofuran, and 3,3-bis(chloromethyl)oxacyclobutane, i.e., monomers polymerizable by cationic catalysts, may not be copolymerized with carbon dioxide with the diethylzinc-water system. The observations described above suggest the anionic nature of the carbon dioxide-epoxide copolymerization with the use of the diethylzinc-water system.

Optical Rotatory Behavior of $R$-(+)-Propylene Oxide-Carbon Dioxide Copolymer

Table II shows the specific optical rotation of the methanol-insoluble copolymer together with data on the homopolymer of $\mathrm{R}$-(+)-propylene oxide for comparison ${ }^{8}$. As in the case of poly-

Table II. Specific optical rotation $[\alpha]_{D}{ }^{25}$ of R-(+)-propylene oxide-carbon dioxide copolymer

\begin{tabular}{lcc}
\hline \multicolumn{1}{c}{ Solvent } & Copolymer $^{\mathrm{a}}$ & $\begin{array}{c}\text { Poly(propylene } \\
\text { oxide) })^{\mathrm{b}, 8}\end{array}$ \\
\hline Benzene & $-14.8\left(-26.2^{\mathrm{c}}\right)$ & -33.3 \\
Chloroform & $-1.4\left(-2.4^{\mathrm{c}}\right)$ & +21.6 \\
\hline
\end{tabular}

a Monomer, $[\alpha]_{D}+13.2$ (neat).

b Monomer, $[\alpha]_{\mathrm{D}}+13.3$ (neat); insoluble part in acetone at $0^{\circ} \mathrm{C}$.

c Value calculated for propylene oxide unit.
(R-propylene oxide), the R-propylene oxidecarbon dioxide copolymer was found to show a remarkable change in optical rotation depending upon the nature of solvent.

Optical rotatory dispersion (ORD) of this R(+)-propylene oxide-carbon dioxide copolymer was measured in benzene and chloroform solutions and analyzed by means of the Drude equation. The results are shown in Figures 1 and 2 .

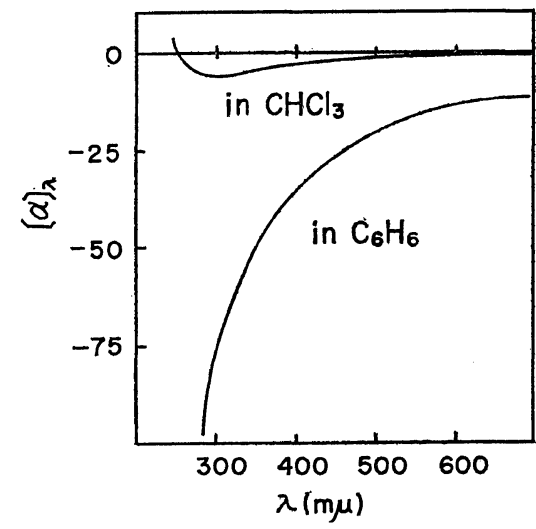

Figure 1. ORD curve of R-(+)-propylene oxidecarbon dioxide copolymer.

(a)

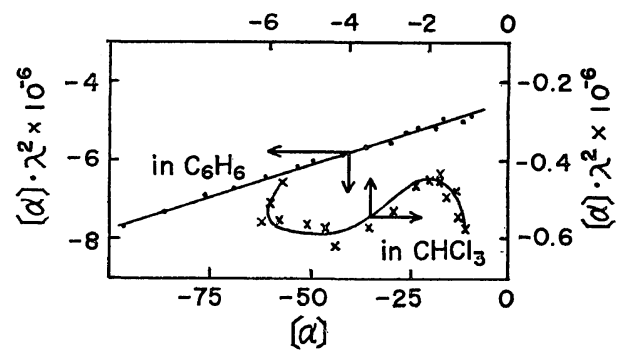

Figure 2. Drude plot of ORD curve of the copolymer.

In the chloroform solution the ORD curve of the copolymer is quite different from that of poly(R-(+)-propylene oxide), reflecting the alternate structure of the copolymer. The ORD curve in chloroform does not obey the one-term Drude equation. On the other hand, the ORD curve in benzene solution is similar to that of poly(R-propylene oxide) except for the magnitude of optical rotation, and it was found to fit the Drude plot as shown in Figure 2. In the case 
of poly(R-propylene oxide) a similar difference in optical rotatory behavior was observed in chloroform and in benzene, probably due to the conformational difference in these solvents ${ }^{8}$.

\section{EXPERIMENTAL}

\section{Materials}

R-(+)-propylene oxide was prepared by Levene's method ${ }^{9}$ and purified by distillation under nitrogen, $[\alpha]_{D}^{25}+13.2^{\circ}$ (neat).

Commercial high purity carbon dioxide gas was introduced into an autoclave directly from a gas cylinder without further purification.

Diethylzinc-water catalyst was prepared under nitrogen atmosphere by the addition of water dropwise into a stirred dioxane solution of diethylzinc $(0.5 \mathrm{~mol} / \mathrm{l})$ cooled by an ice-bath, and subsequent stirring for half an hour at room temperature.

$p$-Nitrobenzoyl chloride was purified by recrystallizing from petroleum ether.

\section{Polymerization}

Copolymerization of $\mathbf{R}-(+)$-propylene oxide with carbon dioxide by diethylzinc-water catalyst $(0.5 \mathrm{~mol} / l$ in dioxane) was carried out by the same pro- cedure as reported previously for the copolymerization of racemic propylene oxide with carbon dioxide ${ }^{1,2}$. The reaction mixture was diluted with benzene, and subjected to freeze-drying. The freeze-dried product was again dissolved in benzene, washed with dilute hydrochloric acid, then with water, and subjected again to freeze-drying. The methanolinsoluble fraction was obtained by precipitating the benzene solution $(150-200 \mathrm{ml})$ of freeze-dried polymer $(1.5-2 \mathrm{~g})$ in methanol $(300-400 \mathrm{ml})$.

\section{Measurement of Optical Rotation}

Optical rotatory dispersion (ORD) measurements were carried out with a Model ORD/UV-5 from the Japan Spectroscopic Company.

Hydrolysis of the Copolymer and Derivation to Propylene Glycol Bis-p-nitrobenzoate

Hydrolysis of the $\mathrm{R}-(+)$-propylene oxidecarbon dioxide copolymer and the isolation of the propylene oxide unit in the form of propylene glycol bis- $p$-nitrobenzoate were carried out by means of the same method we used in treating the copolymer of propylene oxide with 3- phenyl- $\Delta^{4}$-tetrahydrophthalic anhydride, a polyester ${ }^{10}$.

Methanol-insoluble copolymer $(0.70 \mathrm{~g})$ was placed in $40 \mathrm{~m} l$ of $0.5 \mathrm{~N}$ methanolic sodium hydroxide solution and allowed to react overnight with stirring at reflux temperature under nitrogen. Hydrogen chloride gas was bubbled into the reaction mixture at room temperature until the mixture was neutralized, and it was diluted with dioxane and dried with Drierite. After the salt formed and the Drierite was filtered off, the solution was distilled to remove the methanol. Into the residual solution $(c a .15 \mathrm{~m} l$ ) cooled by an ice-bath, pyridine $(7 \mathrm{~m} l)$ and a solution of $p$-nitrobenzoyl chloride $(2.9 \mathrm{~g})$ in dioxane $(15 \mathrm{~m} l)$ were added while stirring. After the mixture was kept standing overnight at room temperature, it was poured into a mixture of ether $(200 \mathrm{ml})$ and water $(70 \mathrm{~m} l)$, and the solution was separated into two layers. The upper layer was washed successively with $1 N$ hydrochloric acid $(50 \mathrm{ml})$, with $1 N$ sodium hydroxide solution $(50 \mathrm{~m} l)$, and twice with water $(50 \mathrm{~m} l)$, then subjected to drying. After the ether was removed, recrystallization of the residual product with ethanol resulted in the formation of cream-colored needles of propylene glycol bis-p-nitrobenzoate $(1.93 \mathrm{~g}$; overall yield, $73 \%) ; \mathrm{mp} 112-119^{\circ} \mathrm{C}$; found: $\mathrm{C}, 54.13 \% ; \mathrm{H}, 3.73 \% ; \mathrm{N}, 7.38 \%\left(\mathrm{C}_{17} \mathrm{H}_{14} \mathrm{O}_{8} \mathrm{~N}_{2}\right.$ requires $\mathrm{C}, 54.44 \% ; \mathrm{H}, 3.77 \% ; \mathrm{N}, 7.48 \%)$. Kinetic resolution in the isolation of the $p$ nitrobenzoate may be ignored since the ester obtained from the $70: 30$ mixture of authentic optically active and racemic samples has an $[\alpha]_{D}$ value identical to the calculated value (Table I, footnote $b$ ).

The authentic sample. R-propylene glycol bis$p$-nitrobenzoate, was prepared by reacting the dioxane solution of $p$-nitrobenzoyl chloride with $\mathrm{R}-(-)$-propylene glycol $\left([\alpha]_{\mathrm{D}}^{25}-15.8^{\circ}\right.$, neat $)$ in the presence of pyridine, $\mathrm{mp} 112-114^{\circ} \mathrm{C}$ (lit. ${ }^{11}$ $\left.107.5-108.5^{\circ} \mathrm{C}\right)$.

\section{REFERENCES}

1. S. Inoue, H. Koinuma, and T. Tsuruta, Polym. Letters, 7, 287 (1969).

2. S. Inoue, H. Koinuma, and T. Tsuruta, Makromol. Chem., 130, 210 (1969).

3. S. Inoue, I. Tsukuma, M. Kawaguchi, and T. Tsuruta, Makromal. Chem., 103, 151 (1967). 
4. S. Inoue, H. Koinuma, Y. Yokoo, and T. Tsuruta, Makromol. Chem., in press.

5. B. Franzus and J. H. Surridge, J. Org. Chem., 31, 4286 (1966).

6. Nan Shieh and C. C. Price, J. Org, Chem., 24, 1169 (1959).

7. C. C. Price and M. Osgan, J. Amer. Chem. Soc., 78, 4787 (1956).

8. T. Hirano, K. Pham Huu, S. Akutsu, and T.
Tsuruta, Preprint, SPSJ 19th Symposium on Macromolecules, Kyoto, 1970, p 677.

9. P. A. Levene and S. Walti, J. Biol. Chem., 68, 415 (1926).

10. K. Matsuura, S. Inoue, Y. Terada, and T. Tsuruta, Kogyo Kagaku Zasshi (J. Chem. Soc. Japan, Chem. Ind. Sect.), 69, 707 (1966).

11. L. H. Klemm and W. Stalik, Tetrahedron, 20, 1667 (1964). 